\title{
Tonometria, paquimetria e comprimento axial ocular em cães glaucomatosos submetidos à ablação uveal intravítrea
}

[Tonometry, pachymetry and globe axial lenght in glaucomatous dogs submitted to intravitreal uveal ablation]

\author{
C.V.S. Brandão ${ }^{1}$, J.L.V. Chiurciu ${ }^{2 *}$, J.J.T. Ranzani ${ }^{1}$, M.J. Mamprim ${ }^{1}$, M. Zanini ${ }^{2}$, J.A. Crocci ${ }^{3}$ \\ ${ }^{1}$ Faculdade de Medicina Veterinária e Zootecnia - UNESP \\ Distrito de Rubião Junior, $\mathrm{s} / \mathrm{n}$ \\ 18618-000 - Botucatu, SP \\ ${ }^{2}$ Aluno de pós-graduação - FMVZ-UNESP - Botucatu, SP \\ ${ }^{3}$ Instituto de Biociências - UNESP - Botucatu, SP
}

\begin{abstract}
RESUMO
Estudaram-se as alterações tonométricas, paquimétricas e de comprimento axial em cães com glaucoma submetidos à ablação uveal intravítrea. Foram avaliados 13 olhos irreversivelmente cegos de cães que apresentavam glaucoma crônico unilateral, nos quais realizou-se a ablação uveal intra-vítrea, por meio de injeção na câmara vítrea de $0,5 \mathrm{ml}$ de sulfato de gentamicina $(40 \mathrm{mg} / \mathrm{ml})$ associado a $0,3 \mathrm{ml}$ de fosfato de dexametasona $(4 \mathrm{mg} / \mathrm{ml})$. As mensurações da pressão intra-ocular (Po), espessura corneana e eixo axial com tonometria de aplanação, paquimetria ultra-sônica e ultra-sonografias modos $\mathrm{A}$ e B foram realizadas no dia da ablação $\left(\mathrm{M}_{0}\right)$ e após uma $\left(\mathrm{M}_{1}\right)$, quatro $\left(\mathrm{M}_{4}\right)$, oito $\left(\mathrm{M}_{8}\right), 12\left(\mathrm{M}_{12}\right), 24\left(\mathrm{M}_{24}\right)$ e 48 semanas $\left(\mathrm{M}_{48}\right)$. A Po diminuiu significativamente em todos os momentos em relação ao $\mathrm{M}_{0}$, com aumento da espessura corneana ao longo do experimento. Nas avaliações ultra-sonográficas notou-se diminuição significativa do bulbo ocular a partir de $\mathrm{M}_{4}$, com medidas ultra-sonográficas modo A significativamente maiores que as do modo B. O procedimento foi efetivo na redução da Po e na diminuição do eixo axial, demonstrando ser viável no controle do glaucoma crônico em olhos irreversivelmente cegos, e ser uma alternativa à enucleação e inserção de prótese ocular.
\end{abstract}

Palavras-chave: cão, ablação farmacológica, glaucoma, tonometria de aplanação, ultra-som

\begin{abstract}
Changes in tonometry, pachymetry and globe axial length in glaucomatous dogs submitted to intravitreal uveal ablation were studied. Thirteen irreversible blind canine eyes were evaluated. They presented unilateral chronic glaucoma, in which intravitreal uveal ablation was performed, through vitreous chamber injection of $0.5 \mathrm{ml}$ of gentamicin sulfate solution $(40 \mathrm{mg} / \mathrm{ml})$ with $0.3 \mathrm{ml}$ of dexametazon dissodic phosphate $(4 \mathrm{mg} / \mathrm{ml})$. The measurements of intraocular pressure (IOP), corneal thickness and globe axial length with aplanation tonometry, ultrasound pachymetry and $A$ and B-mode ultrasound scan were made on the day of the procedure $\left(M_{0}\right)$, and on the next day $\left(M_{1}\right)$, four $\left(M_{4}\right)$, eight $\left(M_{8}\right), 12\left(M_{12}\right), 24\left(M_{24}\right)$ and 48 weeks $\left(M_{48}\right)$ after. The IOP decreased on all moments in relation to $M_{0}$, with corneal thickness increasing throughout the experiment. The ultrasonographic evaluations revealed significant globe decrease from $M_{4}$, with A-scan measurements significantly higher than B-scan. The procedure was effective on intraocular pressure reduction and in shortening the axial length, showing to be feasible to control chronic glaucoma in irreversible blind eyes, as well as an alternative to enucleation and intraocular prothesis.
\end{abstract}

Keywords: dog, pharmacologic ablation, glaucoma, aplanation tonometry, ultrasound

Recebido em 21 de dezembro de 2005

Aceito em 2 de maio de 2007

*Autor para correspondência (corresponding author)

E-mail: j_leandro2000@yahoo.com.br 


\section{INTRODUÇÃO}

O glaucoma canino é definido como uma síndrome em que um ou uma série de eventos levam ao aumento da pressão intra-ocular (Po), em detrimento da manutenção da visão e da saúde do olho (Bedford, 1980; Gelatt, 1991; Martin, 1995). A pressão intra-ocular foi estabelecida como fator de risco primário para o desenvolvimento de neuropatia óptica glaucomatosa no cão (Gelatt e Brooks, 1999), sendo que mudanças degenerativas no nervo óptico e da retina são acompanhadas por deterioração da visão (Ekesten et al., 1997; Whiteman et al., 2002).

Atualmente, a ultra-sonografia é um importante exame complementar em oftalmologia veterinária tanto para determinar a dimensão dos componentes oculares quanto para o diagnóstico de anormalidades de tamanho ocular, tais como glaucoma, phthisis bulbi e microftalmia (Hamidzada e Osuobeni, 1999). Além disso, possibilita detectar presença de hemorragia vítrea, tumores intra-oculares e traumatismos no segmento posterior (Shammas, 1984; ElMaghraby et al., 1995).

A ablação intravítrea com gentamicina é uma técnica já descrita na literatura (Clerc e Robberechts, 1992; Peiffer, 1994), e consiste em procedimento de salvamento de olhos glaucomatosos que apresentem cegueira irreversível, reduzindo a produção de humor aquoso pela destruição parcial do corpo ciliar, com conseqüente diminuição da pressão intraocular e redução do tamanho do bulbo ocular até próximo ao normal, a fim de prover um olho com pouca ou nenhuma necessidade de medicação e menos predisposto a ceratites de exposição.

Os objetivos foram estudar as variações da espessura corneana e pressão intra-ocular após o procedimento de ablação e verificar alterações decorrentes deste no diâmetro axial do bulbo ocular pelos ultra-sons modos A e B, além de comparar as medidas obtidas pelos dois métodos ultra-sonográficos.

\section{MATERIAL E MÉTODOS}

Foram utilizados 13 cães, de raças e idades variadas, machos e fêmeas, portadores de glaucoma crônico unilateral.
Os animais foram submetidos ao exame oftálmico, em ambiente de luminosidade controlada, dos anexos oculares e estruturas do segmento anterior do bulbo ocular, por meio de biomicroscopia em lâmpada de fenda ${ }^{1}$. Avaliações complementares também foram realizadas como o teste de Schirmer ${ }^{2}$ e de fluoresceína sódica ${ }^{3}$. Os que apresentaram alterações oculares ou sistêmicas que inviabilizassem a realização do procedimento de ablação foram descartados.

Foram selecionados cães que apresentavam glaucoma crônico em estágio avançado, com olhos irreversivelmente cegos, de acordo com os seguintes critérios: A - inclusão de animais com acometimento unilateral, observando-se a presença de buftalmia ou hidroftalmia e valores de tonometria de aplanação acima de $33 \mathrm{mmHg}$; B - inclusão de animais com histórico com mais de três semanas de alterações oculares compatíveis com a afecção em estudo; C realização de teste de acuidade visual do olho acometido após a oclusão do olho contralateral não glaucomatoso, e inclusão apenas de cães com deficit visual do olho acometido.

$\mathrm{Na}$ presença de meios oculares, suficientemente transparentes, foram avaliadas, por meio de oftalmoscopia direta, alterações compatíveis com glaucoma crônico no nervo óptico e na retina, tais como disco óptico escavado e de coloração cinza a esbranquiçada e hiperreflexia tapetal com atenuação dos vasos retinianos.

Em ambos os olhos de todos os animais foram realizadas tonometria de aplanação ${ }^{4}$, paquimetria ultra-sônica e ultra-sonografia modos A e B imediatamente antes do procedimento de ablação uveal intravítrea (momento zero - $\mathrm{M}_{0}$ ) e após uma (momento $1-\mathrm{M}_{1}$ ), quatro $\left(\mathrm{M}_{4}\right)$ e oito semanas $\left(\mathrm{M}_{8}\right)$. O tempo de seguimento estendeuse até 12 semanas $\left(\mathrm{M}_{12}\right)$ em 12 animais, 24 semanas $\left(\mathrm{M}_{24}\right)$ em 10 e 48 semanas $\left(\mathrm{M}_{48}\right)$ em cinco.

Após as avaliações iniciais $\left(\mathrm{M}_{0}\right)$, os cães foram pré-anestesiados com cloridrato de

\footnotetext{
${ }^{1}$ Modelo SL-450, Nidek Co. - Japão.

${ }^{2}$ Teste de Schirmer - Ophthalmos - Brasil.

${ }^{3}$ Fluoresceína - Allergan - Brasil.

${ }^{4}$ Modelo Tonopen XL , Mentor - Holanda.
} 
levomepromazina ${ }^{5}$, na dose de $0,5 \mathrm{mg} / \mathrm{kg}$, associado ao butorfanol ${ }^{6}$, na dose de $0,2 \mathrm{mg} / \mathrm{kg}$, via intramuscular. A seguir, foi feita anestesia geral intravenosa de curta duração com propofol $^{7}$, via intravenosa, na dose de $5 \mathrm{mg} / \mathrm{kg}$. No olho acometido por glaucoma crônico, procedeu-se a antissepsia da superfície ocular e do saco conjuntival com solução de polivinilpirrolidona a 1:50 e emprego de pano de campo oftálmico estéril. A seguir, foi introduzida agulha hipodérmica $30 \times 7$ acoplada a uma seringa de $1 \mathrm{ml}$, a seis milímetros posteriormente ao limbo, posicionada entre nove e 10 horas, sendo feita aspiração de cerca de $0,8 \mathrm{ml}$ de humor vítreo. A seringa foi trocada, sendo injetado $0,8 \mathrm{ml}$ de uma solução com $0,5 \mathrm{ml}$ de sulfato de gentamicina $^{8}(20 \mathrm{mg})$ associada a $0,3 \mathrm{ml}$ de dexametasona ${ }^{9}(1,2 \mathrm{mg})$.

Uma injeção subconjuntival de $0,3 \mathrm{ml}$ de gentamicina $(12 \mathrm{mg})$ e $0,3 \mathrm{ml}$ de dexametasona $(1,2 \mathrm{mg})$ foi feita após o procedimento de ablação. Todos os animais foram mantidos com colar tipo elizabetano, sendo prescrito colírio de acetato de prednisolona $1 \%{ }^{10}$, seis vezes ao dia durante 30 dias, e $2,2 \mathrm{mg} / \mathrm{kg}$ de carprofeno ${ }^{11}$, via oral, durante cinco dias.

Nos diferentes momentos de avaliação, mediante instilação prévia do colírio de cloridrato de proximetacaína $0,5 \%{ }^{12}$, foi realizada a aferição da pressão intra-ocular (Po) em ambos os olhos, utilizando tonômetro eletrônico de aplanação. Adotou-se o valor médio após três medidas consecutivas, com coeficiente de variação de $5 \%$. A espessura corneana foi aferida utilizando-se paquimetria ultra-sônica de contato corneano. Após posicionamento perpendicular ao centro da córnea, a aferição foi realizada no olho acometido e contralateral, sendo obtidas cinco medidas consecutivas no modo automático, considerando-se o valor médio dessas mensurações.

A avaliação ultra-sonográfica modo A, denominada ecobiometria, foi realizada

${ }^{5}$ Neozine - Rhodia Pharma - Brasil

${ }^{6}$ Torbugesic - Fort Dodge - Brasil.

${ }^{7}$ Diprivan - Zeneca - Brasil.

${ }^{8}$ Garamicina - Ariston - Brasil

${ }^{9}$ Decadron - Prodome - Brasil.

${ }^{10}$ Pred Fort - Allergan - Brasil.

${ }^{11}$ Rimadyl - Pfizer - Brasil.

${ }^{12}$ Anestalcon - Alcon - Brasil. utilizando-se um ecobiômetro ultra-sônico com transdutor de $10 \mathrm{MHz}^{13}$, no qual foi avaliado o comprimento axial do bulbo ocular. $\mathrm{O}$ ecobiômetro foi utilizado no modo automático com velocidade de $1710 \mathrm{~m} / \mathrm{s}$ para a lente e $1526 \mathrm{~m} / \mathrm{s}$ para as demais estruturas, com o transdutor alinhado ao eixo óptico do olho e em contato com o centro da córnea; foram realizadas 6 medidas consecutivas, obtendo-se o valor médio dessas mensurações.

O ultra-som bidimensional com transdutor mecânico setorial de $7,5 \mathrm{MHz}^{14}$ foi utilizado para a realização da ultra-sonografia modo $\mathrm{B}$ a fím de avaliar-se o comprimento axial do bulbo ocular. Foi utilizada a técnica de contato palpebral, com transdutor mantendo-se alinhado ao eixo óptico do olho. Os exames foram realizados sempre pelo mesmo examinador.

Para a avaliação das variáveis tonométricas, paquimétricas e ultra-sonográficas, foi utilizada a análise multivariada de perfil com nível de significância de 5\% de probabilidade (Morrison, 1990) pela qual foi comparado o efeito do tratamento entre os vários momentos, bem como entre os olhos acometidos e normais em cada momento avaliado. Os métodos de medida do eixo axial do bulbo ocular, ultra-som modos A e $\mathrm{B}$, foram comparados pelo teste $\mathrm{t}$ de Student pareado, com nível de significância de 5\% (Morrison, 1990), utilizando as médias das medidas de ambos os olhos.

\section{RESULTADOS E DISCUSSÃO}

Com relação à pressão intra-ocular (Fig. 1), o olho acometido apresentou, em média, valor significativamente maior antes da ablação intravítrea $\left(\mathrm{M}_{0}\right)$ em comparação com os valores médios de Po em todos os momentos após o procedimento, confirmando os resultados de Bingaman et al. (1994), bem como quando comparado ao olho normal no momento $\mathrm{M}_{0}$. Não houve diferença significativa dos valores médios de Po entre os momentos no olho normal.

A redução da Po no $\mathrm{M}_{1}$ e $\mathrm{M}_{4}$ foi, em média, de $73,6 \%$, valor semelhante ao verificado por Moller et al. (1986), que após um mês de ablação

\footnotetext{
${ }^{13}$ Modelo US-800, Nidek Co. - Japão.

${ }^{14}$ ModeloEUB 405, Hitachi - Japão.
} 
obtiveram 76,3\% de diminuição da Po. Segundo esses autores, dos 13 olhos avaliados, sete apresentaram Po com valores de 18 a $21 \mathrm{mmHg}$, quatro variaram entre 10 a $18 \mathrm{mmHg}$ e dois com Po abaixo de $6 \mathrm{mmHg}$ com um mês de tratamento, enquanto no presente estudo, no mesmo tempo de seguimento, 10 olhos apresentaram Po abaixo de $10 \mathrm{mmHg}$ e três, acima de 30mmHg (Moller et al., 1986). A diminuição dos valores, em média, da Po a partir de oito semanas até o final do experimento foi de $84,3 \%$, revelando a necessidade de maior tempo de acompanhamento para se estimar sua real redução nos olhos submetidos à ablação intravítrea.

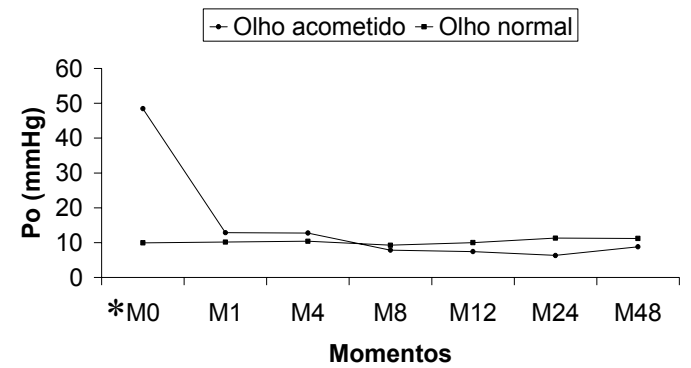

Figura 1. Valores médios da pressão intra-ocular (Po) dos olhos acometidos e normais de cães nos diferentes momentos de avaliação.

*Diferença significativa entre os olhos acometidos e normais $(\mathrm{P}<0,05)$.

Observaram-se valores de espessura corneana significativamente maiores nos olhos acometidos em relação aos contralaterais, decorrentes do edema estromal pela alteração dos mecanismos de "bomba" do endotélio corneano (Gelatt e Brooks, 1999), (Fig. 2). Embora a espessura corneana aumentada possa interferir na acurácia da mensuração da Po por tonometria da aplanação, superestimando seu valor (Copt et al., 1999), sua influência não foi significativa nas medidas de Po deste experimento, por se tratar de cães com glaucoma crônico, com pressões intra-oculares bem acima do normal. No olho acometido, observou-se aumento da espessura corneana, com valores médios ao redor de $1100 \mu \mathrm{m}$ no $\mathrm{M}_{12}$ e $\mathrm{M}_{24}$, significativamente maiores que o valor médio obtido no olho normal em $\mathrm{M}_{0}$, ou seja, $620 \mu \mathrm{m}$.

A manutenção do aumento da espessura corneana, a despeito da diminuição da Po, pode ser explicada pela perda de células endoteliais causada pelo glaucoma inicial e pela diminuição do aporte de nutrientes a essas células decorrente da diminuição quantitativa de humor aquoso. Há aumento da demanda da metabólica nas células endoteliais remanescentes, mas insuficientes para manter a turgescência corneana, resultando em edema corneano permanente (Gelatt e Brooks, 1999).

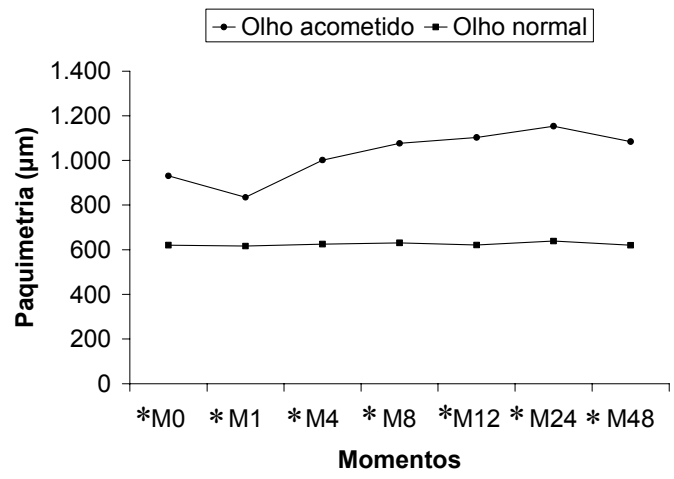

Figura 2. Valores médios da espessura corneana dos olhos acometidos e normais de cães nos diferentes momentos de avaliação.

*Diferença significativa entre os olhos acometidos e normais $(\mathrm{P}<0,05)$.

Nas avaliações de comprimento axial do bulbo ocular, obtidos por ecobiometria (Fig. 3) e ultrasonografia modo B (Fig. 4), observaram-se valores médios mais alto, no olho acometido em relação ao olho contralateral em $\mathrm{M}_{0}$ e $\mathrm{M}_{1}$, confirmando a presença de buftalmia secundária ao glaucoma. Em ambos os modos de ultra-som do olho acometido, houve redução progressiva do comprimento axial ao longo dos momentos: de $M_{4}$ a $M_{12}$ essa redução foi de $12,9 \%$ nos valores obtidos por ecobiometria e $15,6 \%$ nos obtidos por ultra-som modo $\mathrm{B}$, e a partir do $\mathrm{M}_{24}$, a redução foi de 21,3 e 20,4\%, respectivamente. $\mathrm{Na}$ literatura veterinária consultada, não foram encontrados dados referentes ao comprimento axial em animais glaucomatosos submetidos à ablação.

Nos dois modos de ultra-som, verificaram-se valores de comprimento axial do olho acometido estatisticamente semelhantes, em média, ao olho contralateral a partir do $\mathrm{M}_{4}$; entretanto constatouse clinicamente presença de phthisis bulbi em cinco animais, com valores de comprimento axial abaixo do valor médio de 20,503 e $19,187 \mathrm{~mm}$, obtidos por ecobiometria e ultra-som modo $\mathrm{B}$, respectivamente, dos olhos normais, observação compatível com os resultados de Bingaman et al. (1994). 


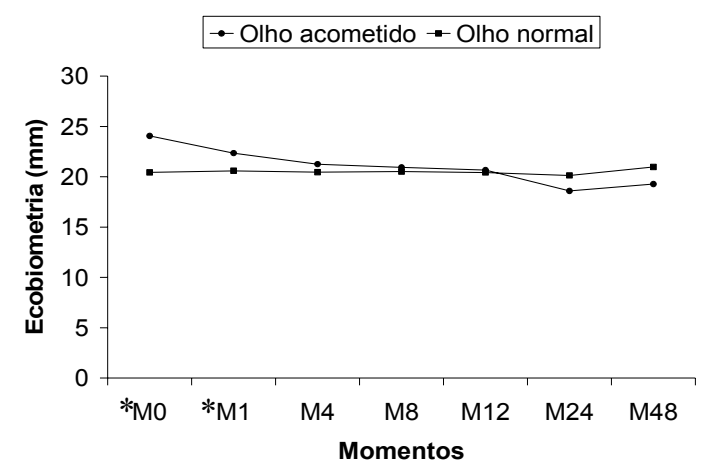

Figura 3. Valores médios do comprimento axial obtido por ecobiometria dos olhos acometidos e normais de cães nos diferentes momentos de avaliação.

*Diferença significativa entre os olhos acometidos e normais $(\mathrm{P}<0,05)$

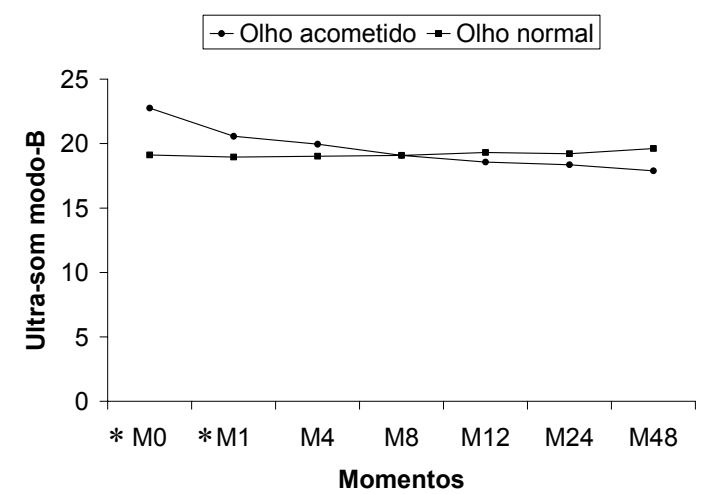

Figura 4. Valores médios do comprimento axial obtido por ultra-sonografia modo B dos olhos acometidos e normais de cães nos diferentes momentos de avaliação.

*Diferença significativa entre os olhos acometidos e normais $(\mathrm{P}<0,05)$.

Os valores do comprimento axial obtidos por ecobiometria e por ultra-sonografia modo B mostram que houve, em média, diferença significativa entre eles, de aproximadamente $2 \mathrm{~mm}$ (Tab. 1), isto é, os valores ecobiométricos foram mais altos. Pelo gráfico de dispersão em relação à reta $\mathrm{Y}=\mathrm{X}$ (Fig. 5), as medidas obtidas pelo ecobiômetro são, na maioria das vezes, maiores que as reveladas pelo ultra-som modo B, e a correlação de concordância foi de 0,805 (Zar, 1996). Essa correlação indica que, apesar de ter havido similaridade positiva, ela não foi tão evidente quanto a encontrada por Laub e Ingrisch (1984), no estudo com ventrículos laterais em recém-nascidos, de 0,945 , o que explica a diferença significativa entre ecobiometria e ultrasom modo B.

Tabela 1. Valores médios, em mm, e desviopadrão de ecobiometria e ultra-sonografia modo $\mathrm{B}$, dos olhos acometidos e normais de cães

\begin{tabular}{lcc}
\multicolumn{1}{c}{ Método } & $\begin{array}{c}\text { Média do } \\
\text { comprimento } \\
\text { axial }(\mathrm{mm})\end{array}$ & Desvio-padrão \\
\hline Ecobiometria & $21,406 \mathrm{a}$ & 3,366 \\
Ultra-som modo B & $19,859 \mathrm{~b}$ & 3,455 \\
\hline Letras distintas na coluna indicam & diferenças \\
significativas entre os valores $(\mathrm{P}<0,05)$. &
\end{tabular}

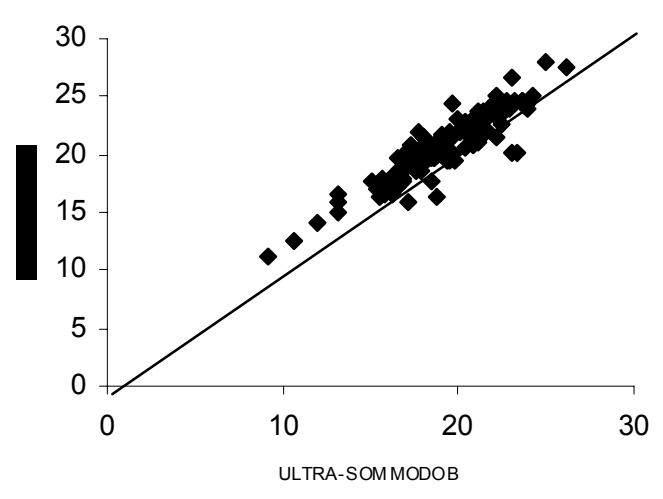

Figura 5. Dispersão dos valores de ecobiometria e ultra-sonografia modoB em relação à reta $\mathrm{Y}=\mathrm{X}$, em olhos de cães.

Cottrill et al. (1989) compararam o comprimento axial dos olhos de cães, mesocefálicos e dolicocefálicos, por ultra-som modos A e B, utilizando transdutores de $10 \mathrm{MHz}$, com mensurações físicas diretas após a eutanásia, e não verificaram diferença significativa entre os valores. El-Maghraby et al. (1995), ao utilizarem olhos de ovinos e bovinos, também não observaram diferença significativa entre os métodos ultra-sonográficos de medida do comprimento axial do bulbo ocular. Ekesten (1994) realizou medidas oculares no modo A e modo B e verificou que o comprimento axial foi significativamente menor no modo $B$ em relação ao modo $\mathrm{A}$, atribuído à maior sensibilidade desse método à força aplicada sobre o olho durante o exame. $\mathrm{O}$ autor relatou maior eficiência do ultrasom modo A e enfatizou que uma ligeira sedação dos animais é vantajosa por aliviar o estresse e facilitar a realização do exame. Hamidzada e Osuobeni (1999) também observaram, em olhos de camelos, valores subestimados do 
comprimento axial do bulbo do modo $\mathrm{B}$ em relação ao modo $\mathrm{A}$, assim como Brandão et al. (2004), em olhos de ovinos.

Segundo Hamidzada e Osuobeni (1999), a diferença entre os métodos ultra-sonográficos pode ser devida à diferença na velocidade de propagação do som através dos diferentes tecidos oculares. De acordo com esses autores, a diferença da velocidade de refração entre cristalino, a qual apresenta-se maior, e humor vítreo faz com que a imagem ultra-sonográfica apareça anteriormente, subestimando a medida. Essa diferença de propagação está presente em ambos os métodos ultra-sonográficos, mas é mais perceptível no modo B. Além disso, Cottrill et al. (1989) relataram que a técnica de contato palpebral, utilizada na mensuração das medidas ultra-sonográficas modo $\mathrm{B}$, pode levar à indentação do bulbo ocular pelo transdutor, subestimando o comprimento axial.

\section{CONCLUSÕES}

O procedimento de ablação uveal intravítrea foi efetivo na redução da pressão intra-ocular do olho com glaucoma e induziu neles aumento da espessura corneana. A diminuição progressiva do comprimento axial após o procedimento indica redução efetiva do tamanho do bulbo ocular. Os métodos ultra-sônicos apresentaram concordância positiva das medidas de comprimento axial, ambos demonstrando eficiência nas mensurações, apesar da diferença significativa entre eles. A ablação uveal intravítrea com gentamicina mostrou ser um procedimento viável para o controle do glaucoma crônico em olhos irreversivelmente cegos, apresentando-se como alternativa à enucleação e inserção de prótese ocular.

\section{REFERÊNCIAS BIBLIOGRÁFICAS}

BEDFORD, P.G. The aetiology of canine glaucoma. Vet. Rec., v.107, p.76-82, 1980.

BINGAMAN, D.P.; LINDLEY, D.M.; GLICKMAN, N.W. et al. Intraocular gentamicin and glaucoma: a retrospective study of $60 \mathrm{dog}$ and cat eyes (1985-1993). Vet. Comp. Ophthalmol., v.4, p.113-119, 1994.

BRANDÃO, C.V.S.; CHIURCIU, J.L.V.; RANZANI, J.J.T. et al. Comparação entre ultra-sonografia modo A, modo B e medidas diretas em olhos de ovinos. Braz. J. Vet. Res. Anim. Sci., v.41, supl., p. 68-69, 2004.
CLERC, B.; ROBBERECHTS, C. Treatment of absolute glaucoma by intravitreous injection of gentamicin. Clinical research report. Recueil Med. Vet., v.168, p.97-103, 1992.

COPT, R.P.; THOMAS, R.; MERMOUD, A. Corneal thickness in ocular hypertension, primary open-angle glaucoma, and normal tension glaucoma. Arch. Ophthalmol. v.117, p.14-16, 1999.

COTTRILL, N.B.; BANKS, W.J.; PECHMAN, R.D. Ultrasonographic and biometric evaluation of the eye and orbit of dogs. Am. J. Vet. Res., v.50, p.898-903, 1989.

EKESTEN, B. Biological variability and measurement error variability in ocular biometry in Samoyed dogs. Acta Vet. Scand., v.35, p.427-433, 1994.

EKESTEN, B.; BJERKAS, E.; KONGSENGEN, K. et al. Primary glaucoma in the Norwegian elkhound. Vet. Comp. Ophthalmol., v.7, p.14-18, 1997.

EL-MAGHRABY, H.M.; NYLAND, T.G.; BELLHORN, R.W. Ultrasonographic and biometric evaluation of sheep and cattle eyes. Vet. Radiol. Ultrasound, v.36, p.148-151, 1995.

GELATT, K.N. The canine glaucomas. In: . Veterinary ophthalmology. 2.ed. Philadelphia: Lea \& Febiger, 1991. p.396-427.

GELATT, K.N.; BROOKS, D.E. The canine glaucoma. In. GELATT, K.N. (Ed). Veterinary Ophthalmology. 3. ed. Philadelphia: Lippincott Williams \& Wilkins, 1999. p.701754.

HAMIDZADA, W.A.; OSUOBENI, E.P. Agreement between A-mode and B-mode ultrasonography in the measurament of ocular distances. Vet. Radiol. Ultrasound, v.40, p.502-507, 1999.

LAUB, M.C.; INGRISCH, H. Systematic ultrasound imaging of the lateral ventricles. A-scan versus B-scan. Monatsschr Kinderheilkd, v.132, p.286-289, 1984.

MARTIN, C. L. Glaucoma. In: SLATTER, D. (Ed). Manual de cirurgia de pequenos animais. 2.ed. São Paulo: Manole, 1995. p.1506-1521.

MOLLER, I.; COOK, C.S.; PEIFFER Jr., R.L. et al. Indications for and complications of pharmacological ablation of the ciliary body for the treatment of chronic glaucoma in the dog. J. Am. Anim. Hosp. Assoc., v.22, p.319326, 1986.

MORRISON, D.F. Multivariate statistical methods. São Paulo: Mc Graw - Hill, 1990. 450p.

PEIFFER, R.L. Intraocular gentamicin in glaucoma. Vet. Comp. Ophthalmol., v.4, p.166, 1994.

SHAMMAS, H.J. Atlas of ophthalmic ultrasonography and biometry. St. Louis: Mosby, 1984. 321p.

WHITEMAN, A.L.; KLAUSS, G.; MILLER, P.E. et al. Morphologic features of degeneration and cell death in the neurosensory retina in dogs with primary angle-closure glaucoma. Am. J. Vet. Res., v.63, p.257-261, 2002.

ZAR, J.H. Biostatistical analysis. New Jersey: Prentice-Hall, 1996. 718p 